\title{
Stress and Coping Mechanisms among nursing students in Kathmandu
}

\author{
Bista $\mathrm{B}^{1}$, Bhattrai $\mathrm{B}^{2}$ and Khadka $\mathrm{N}^{3}$ \\ 1,2,3 Department of Nursing
}

\section{Abstract:}

Background: Every person experiences different forms of stress throughout their life. Therefore a student nurse is no exception as they have to adjust to an entirely new environment on joining nursing. Stress in nursing students is an area of growing concern. The stress among the students can have serious effects on their health and studies. It may affect in psychological distress, physical complains, behavior problems and poor academic performance.

\section{Objective}

The main objective of this study was to assess the level of stress and coping mechanisms among nursing students.

\section{Methodology}

A descriptive cross-sectional study was done among 283 nursing students of Manmohan Memorial Institute of Health Sciences (MMIHS), by using purposive sampling technique. The level of stress was assessed using Perceived stress scale-14 (PSS-14) and coping mechanism was assessed using Brief cope inventory. The data analysis was done using SPSS version 20. Descriptive and inferential statistics was used and Chi-square test was applied for association.

\section{Result}

The results were found that $61.5 \%$ of the respondents have moderate stress and $27.9 \%$ of the respondents have severe stress and mild stress only by $10.6 \%$. Likewise $44.5 \%$ of the respondents were very often stressed due to test, examination and evaluations where as half of the majority of respondents were some time deal with dying or seriously ill patient. Similarly $58.3 \%$ used Emotion-focused strategies for coping mechanism and $3.9 \%$ of the respondents used Problem-focused strategies for coping mechanism. 


\section{Conclusion}

Majority of the nursing students were found to have moderate stress. Perceived stress scale was found to be statistically significant with age, educational status of the respondents, education of father and mother. Majority of the respondents used emotion-focused strategies for coping and minority of the respondents used problem-focused coping strategies

\section{Key word : stress factors, perceived stress, coping strategies}

\section{Introduction:}

Stress is a way of body's response to any kind of demand. Coping is the process of managing demands that are considered as challenging to the individual. Stress in nursing students can be triggered from a variety of issues including academics, and financial concerns According to nursing curriculum they must learn theory as well as practical classes at the sametime. They face different stressors can be related with academic and clinical as well. Stress affects physically, mentally, academically. They may feel sad, nervousness, sleeplessness, loneliness etc. Stress may affect the students' health and their academic performance. However, students experience increased tension prior to their clinical rotation and written examination especially their finals. In nursing, students experience stress in day to day life

Clinical sources of stress include working with dying patient, interpersonal conflict with other nurses, insecurity about clinical competence and fear of failure and interpersonal relations with patients, work overload and are concerned about nursing care given to the patient. Other potential sources of stress are assignment submission, excessive homework, assessment deadlines, unclear assignments, uncomfortable classrooms and relations with faculty members. (1)

There are some ways of stress management among the student nurses in their studies and their clinical areas i.e. family brings great support mentally and emotionally. During problem they need to talk to family members who are always there to help. Social support networks can also help the students during problem or stress. Developing a positive optimistic attitude can help them to cope more effectively with stress. Although students cannot avoid these stressors, their ability to adjust to demands and cope with these stressors is important in achieving success in the college academic and social environment. (2) 
Studies comparing the stress levels of various professional students found that nursing students experiences higher levels of stress than medical, social work and pharmacy students. Rella, Win wood and Lushington (2008) found that up to $20 \%$ of nursing graduates were reporting serious unmanaged fatigue and stress during their student life. (3)

In a study done at Private Nursing college, Punjab findings revealed that out 180 nursing students $62(34.4 \%)$ had moderate stress whereas $59(32.8 \%)$ and $59(32.8 \%)$ fall in the category of low and high stress respectively. (4)

The identification of the stressors and about the perceived stress by the nursing students can help for better assessment of effective coping strategies to overcome stress. This can further help to minimize the stressors that are avoidable and guide the students for better future.

\section{Methodology:}

Descriptive, Cross-sectional study was used to identify the perceived stress level and coping mechanisms among nursing students. The Study was conducted at MMHSS, Soalteemode, Kathmandu. on-probability Purposive sampling technique was used. The sample size was 283. (With 0\% non-respondents). Structured questionnaire was developed with Cohen Perceived Stress Scale by Sheldon Cohen for measuring perceived stress level and Brief cope inventory by Carver et al. for coping mechanisms. Self-administered questionnaires were distributed to the respondents with clear instructions. Computer software SPSS version 20 was used for data processing and analysis.

The self-administered questionnaires were made in simple language as far as possible to ensure validity.Pre-testing was done with the questionnaire in $10 \%$ of total sample population. Necessary modifications were done after pretesting. Approval from the IRC of the campus was taken. Formal permission was taken from each respondent. Informed consent was taken from each respondent. The purpose and objective of study was informed. Anonymity and confidentiality of the respondents was maintained.

\section{Results:}

Regarding to Socio-demographic characteristics half of the majority (54.8\%) were in the age group less than20. Level of education in PCL, Bsc Nursing \&BNS were represent 42\% 35\% \&23\% respectively. Similarly more than half of majority respondents $(59 \%)$ father had have higher 


\section{Original Article}

secondary education where as only $44.9 \%$ mother' education were secondary level of education. A very few percentage of parents were illiterate. About income, $39 \%$ had monthly family income between 40 to 60 thousands and $3.2 \%$ had less than 20,000 .

In regarding to type of residence $88 \%$ of the respondents were living outside the hostel, similarly travel from residence to college, $31.4 \%$ took more than 50 mins followed by $18.4 \%$ travel 40 to 50 mins and $17 \%$ spend only $<20 \mathrm{~min}$. Availability of play and recreational activities $58.7 \%$ of the respondents had availability of play and recreational activities and $41.3 \%$ did not have availability of play and recreational activities.

Hours available for play and recreational activities $49.3 \%$ had less than $2 \mathrm{hrs}, 36.2 \%$ had $2-4 \mathrm{hrs}$ and $14.5 \%$ had more than 5 hrs for play and recreational activities.

Regarding the stress factors associated in theory as well as practice, half of the majority of respondents $(54.8 \%)$ some time had to deal with dying or seriously ill patients. $49.5 \%$ sometimes felt that they were given excessive assignment, $46.6 \%$ sometimes felt theory and practice gap, Similarly very often had been stressed due to stressed due to to test, examination and evaluation by $44.5 \%$, similarly assignment dead line by $39.2 \%$.

Relationship with peers, clinical supervisor and nursing staffs ,77.7\% respondents had good relationship with peers, clinical supervisor and nursing staff where as poor relationship followed by22.3\% respondents. However $76.2 \%$ of the respondents were stressed due to poor relationship with peers, clinical supervisor and nursing staffs

According perceived stress $61.5 \%$ respondents have moderate stress where as $10.6 \%$ have only mild stress. Similarly coping strategies during stress, $58.3 \%$ respondents used emotion-focused coping strategies, and $37.8 \%$ used dysfunctional coping strategies.3.9\% used problem-focused strategies

Association between selected variables and perceived stress level shows that age, educational status of the respondents, education of their father and mother are statistically significant with perceived stress level $(p<0.05)$ whereas there is no significance on type of family, monthly family income, time to travel and availability of play and recreational activities. 


\section{Original Article}

\section{Discussion:}

Stress in nursing students is an area of growing concern. The stress among the students can have serious effect on health and studies. The present study was conducted to assess stress level and factors associated with stress among nursing students and use of their coping strategy.

The present study shows that among 283 respondents $61.5 \%$ of the respondents had moderate stress and $27.9 \%$ of the respondents had severe stress. This finding is consistent with the findings of previous studies a study conducted by (4) that out of 180 nursing students $34.4 \%$ had moderate stress whereas 32.8\% had high stress respectively. Another study by (2) reported that two third of the students had moderate stress $(63.6 \%)$ whereas 23 per cent students had severe stress.

Regarding socio-demographic findings in the present study,perceived stress level was found to be more in nursing students of age less than 20 than in age group more than 20 . So, the nursing students of age group less than 20 might perceive stress more than those of age group more than 20 due to lack of experiences and responsibilities.

The perceived stress by the level of nursing students was found to be more in the B.Sc. Nursing students than PCL nursing and BNS. It might be because in comparison to PCL Nursing the curriculum followed by B.Sc. Nursing students are much more complex and they have more workload than PCL Nursing and in comparison to BNS, the B.Sc. Nursing are new to nursing and the clinical exposures. The findings of the present study thus indicate to us how important of curriculum, clinical exposure in multi concerned hospitals and community are and the need to focus counseling on them.

The present study identified various factors causing stress in nursing students. The study shows that age, educational status of the respondents, education of father and mother of the respondents are the major contributing for stress. A similar study of (4) shows that among the factors contributing to stress, the environmental factors(40\%), interpersonal factors (30\%) and academic factors had only $19 \%$ contribution.।

Among the academic and clinical factor $44.5 \%$ of the respondents were stressed due to test, examination and evaluations and $10.2 \%$ of the respondents were stressed because they have to deal with dying or seriously ill patient .It is however partly consistent with (3) reported the common sources of clinical stress among nursing students were the preparation and submission of the 


\section{Original Article}

assignment followed by difference between real situation and ideal situation (theory practice gap), lack of hospital facilities and equipment for procedures patient with serious diseases suffering and unfamiliar environment of the health care setting, doing bedside care to the patient, little direction, to what expected from teacher, to many patients to handle, inadequate knowledge and skill needed to care the patient, relationship with the clinical instructors/supervisors.

In contrast, a study done by Anand,S.et.al, 2015 reported that the dissatisfaction with the facilities in the dormitory were a major environmental stressor among majority of the students as they reported overcrowding in the study area (48.7\%), inadequate water supply (35.7\%) and overcrowding in the room (33.7\%) (5). Another study done by Ajibade, B.L.et.al, 2016 reported that the major stress factors is their economic status rather that personal and academic factors. It might be because the students abroad are independent and more used to sedentary lifestyle. But in our context the students are dependent on parents and do not face economic problems as that of the students abroad (6).

In the present study, the respondents were found to use different coping strategies to cope with stress. Majority of the respondents $58.3 \%$ used Emotion-focused strategies for coping mechanism and $3.9 \%$ of the respondents used Problem-focused strategies for coping mechanism. The nursing students were found to use emotional support, humor, positive reframing, acceptance, self-distraction, behavioral disengagement mostly rather than planning, substance use and denial. It might be because it is easy to be cope using emotional strategies than problem based strategies for their comfort.

This finding is consistent with the findings of previous studies a study conducted by Rajesh Kumar, Nancy reported that most commonly used strategy by the students was ventilating feelings as complaining to their friends (36.7\%) and crying (22.8\%), listen to music, stereo or radio (36.7\%), engaged in day-dreaming (23.3\%) hobby (14.1\%) eat food (13.9\%) try to think of or see good things $(26.7 \%)$, try to organize their life (19.4\%) and try to make their own decisions (18.3\%). Around $32 \%$ of the students tried to cope with the stress by helping other people in solving their problems or by blaming others for their problems respectively (4).

The present study reflects that the major sources of stress among nursing students are the clinical and academic factors. Younger students tend to experience emotional symptoms as a response to stress compared to that older students. This may be due to fact that as students get older, they 


\section{Original Article}

gained not only better knowledge and clinical expertise but also with problem solving skills and stress preventive strategies necessary when faced with various stressors. Every nursing students face stress in day to day life and it cannot be ignored (7). Moderately students are able to cope up with various stressors faced during their nursing education. Rather a better approach is needed to cope with the stress so that the nursing student can be healthy mentally also. This results information is also useful for nursing management and nursing teachers to identifying students' needs, effective planning, facilitating their learning in the academic and clinical setting, to reduce or prevent stress during their education and training.

\section{Conclusion:}

It has been concluded that majority of the nursing students had felt moderate stress due to academic, clinical and interpersonal relationship with peers, staffs and supervisors. Similarly two third majorities have stressed due to poor relationship with others It was found then the perceived stress level was statistically significant with age, educational status, education of father and mother. Further, the nursing students were found to use emotional-focused strategies for coping rather than problem-focused and dysfunctional coping strategies.

They use different coping strategies to overcome stress so as to maintain a balance on their physical and mental health. Coping with stress for a student nurse is a dynamic. It is important to find out the sources of stress and coping strategies used by the students so that they can be helped to cope well with upcoming problems and situations.

\section{Bibliography}

1. DR, WI, DK. A descriptive study to assess the causes of stress and coping strategies used by the newly admitted basic B.Sc nursing students. Nursing and midwifery research journal. 2009;: p. 31-37.

2. CS, SS, RKS. Level of stress and coping strategies used by nursing interns. Nursing and Midwifery Research Journal. 2011;: p. 152-160.

3. Shrestha T. Stress among PCL Nursing students of TUIOM Nursing campuses in the Kathmandu Valley. Journal of Institute of medicine. 2013; 35(3): p. 56-61. 


\section{Original Article}

4. Rajesh kumar N. Stress and Coping Strategies among Nursing Students. Nursing and Midwifery Research Journal. 2011;: p. 142.

5. Singh C SR. Level of Stress and Coping strategies by nursing interns. Nursing and Midwifery Journal. 2011; 7(4): p. 152-160.

7. J R. A comparative study of professional students stress. Journal of Dental Education. 2009; 73(3): p. 328-337.

8. Gibbons C DM,MM. Stress and Eustress in Nursing Students. Journal of Advanced nursing. 2008; 61(3). 